\title{
Cardiac MRI: Luxury or Necessity, beyond the Electrocardiogram and Biology in the Management of Acute Coronary Syndrome in Young Patients? About 2 Cases Reports in Sub-Saharan Environment
}

\author{
Simon Antoine Sarr ${ }^{*}$, Kana Babaka1, Fatou Aw ${ }^{1}$, Malick Bodian', Joseph Mingou', \\ Mouhamadou Bamba Ndiaye1, Mor Beye1, Amalia Owona', Babacar Mbengue', \\ Adama Kane', Maboury Diao', Serigne Abdou Ba ${ }^{1}$ \\ ${ }^{1}$ Cardiology Department, Teaching Hospital Aristide Le Dantec, Dakar, Sénégal \\ ${ }^{2}$ Radiology Department, Hospital Principal, Dakar, Sénégal \\ Email: *sarrsimantoine@yahoo.fr
}

How to cite this paper: Sarr, S.A., Babaka, K., Aw, F., Bodian, M., Mingou, J., Ndiaye, M.B., Beye, M., Owona, A., Mbengue, B., Kane, A., Diao, M. and Ba, S.A. (2018) Cardiac MRI: Luxury or Necessity, beyond the Electrocardiogram and Biology in the Management of Acute Coronary Syndrome in Young Patients? About 2 Cases Reports in Sub-Saharan Environment. World Journal of Cardiovascular Diseases, 8, 102-110. https://doi.org/10.4236/wjcd.2018.82010

Received: December 10, 2017

Accepted: January 30, 2018

Published: February 2, 2018

Copyright (c) 2018 by authors and Scientific Research Publishing Inc. This work is licensed under the Creative Commons Attribution International License (CC BY 4.0).

http://creativecommons.org/licenses/by/4.0/ c) (i) Open Access

\begin{abstract}
Introduction: Precordial pain is a common reason for admission in cardiology, and has many causes. Acute myocarditis in its pseudo-infarctoid form is sometimes difficult to differentiate from myocardial infarction. Cardiac magnetic resonance imaging (MRI) helps to differentiate these two disease entities. We report the respective cases of two young patients, one presenting with myocarditis whilst the other with myocardial infarction. Case Report: We present the cases of two patients. The first who had a recent history of febrile syndrome is a 23-year-old who stopped smoking 3 months prior to presentation whilst the second is a 22-year-old professional footballer with a history of stress with no other cardiovascular risk factors. They were respectively admitted in our emergency department for a constrictive, intense chest pain. Physical examination was normal. The chest pain in both patients was associated with elevated cardiac markers, primary repolarisation abnormalities on ECG, wall motion abnormalities as well as left ventricular systolic dysfunction on transthoracic echocardiography. Coronary angiograms were normal in both patients. In the first patient, MRI concluded with an acute myocarditis with apical akinesia extending to the anterior wall, a T2 hypersignal indicative of myocardial edema, and uptake of a nodular heterogeneous contrast without affecting the sub-endocardial layers on the late enhancement sequences. In the second patient, MRI showed an appearance consistent with acute extensive
\end{abstract}


infarction in the antero-apical region with severe hypokinesia and late quasi-transmural enhancement, impairment of the anterior papillary muscle of the mitral valve and a reduced left ventricular ejection fraction at $33 \%$. In addition to analgesics, the first patient was treated with perindopril and bisoprolol, and the second patient received antithrombotic and anticoagulant treatment. There was clinical improvement in both patients. Conclusion: Cardiac MRI is a useful diagnostic tool for the precise diagnosis of precordial pain with elevated cardiac enzymes, especially in young patients.

\section{Keywords}

MRI, Myocardial Infarction, Myocarditis, Young Patient

\section{Introduction}

Precordial pain is a common reason for admission in cardiology. Its causes are numerous including myocardial infarction which is difficult to differentiate from myocarditis in its pseudo-infarctoid form. Both disease entities may present as precordial pain with elevation of cardiac enzymes and ECG repolarization abnormalities [1]. Differential diagnosis is often challenging especially in young people. In this context, the role of cardiac magnetic resonance imaging (MRI) is paramount, making it possible to obtain a precise etiology. We present two cases demonstrating the value of cardiac MRI: the first a 23-year-old patient with myocarditis and another, a 22-year-old patient with myocardial infarction.

\section{Case Reports}

Case 1: A 23-year-old patient, an ex-smoker who stopped three months prior to presentation, having no other known cardiovascular risk factor, no history of cocaine or other stimulant consumption. He has a recent history of febrile episode associated with vomiting a week prior to hospitalization. He was admitted for a centrally located chest pain radiating to the upper limbs. The pain was constrictive, very intense, and started seven hours prior to presentation. His general condition was satisfactory with a normal blood pressure at $120 / 80 \mathrm{mmHg}$, heart rate at 97 beats per minute, respiratory rate at 25 cycles per minute and temperature at $36.5^{\circ} \mathrm{C}$. Cardiovascular and respiratory systems examination were normal. There were no distended jugular veins or edema of the lower limbs. Laboratory analyses showed elevated troponin I level at $2.38 \mathrm{ng} / \mathrm{l}$ (normal $<0.3$ $\mathrm{ng} / \mathrm{l})$, a hypochromic microcytic anemia at $7.9 \mathrm{~g} / \mathrm{dl}$, raised inflammatory markers with fibrinemia and C-reactive protein respectively at $5.13 \mathrm{~g} / \mathrm{l}$ and $24 \mathrm{mg} / \mathrm{l}$. Fasting blood glucose, lipid profile and renal function were all normal. The electrocardiogram inscribed a regular sinus rhythm with a heart rate at 92 cycles per minute, an extensive anterior sub-epicardial lesion with a mirror image in inferior image (Figure 1). The chest X-ray was normal. Transthoracic Doppler echocardiography showed septo-medial and septo-apical hypokinesia of the left 


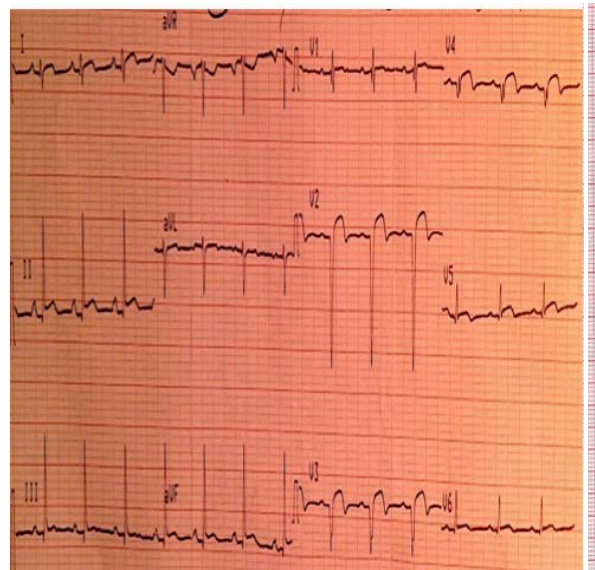

(a)

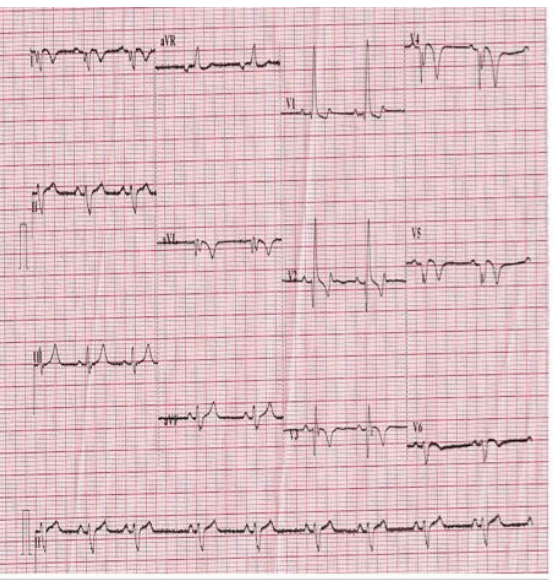

(b)

Figure 1. Electrocardiogram at admission of the two patients. (a) ST elevation in extended anterior leads (V2 to V6, DI, aVL) with reciprocal ST depression in inferior leads; (b) ST elevation in apical and latéral leads (V4, V5, V6) + anterior extensive necrosis (V1 to V6, DI, aVL) + complete right bundle branch block + isolated ventricular extrasystole.

ventricle with moderate reduction of the left ventricular ejection fraction at $39 \%$. The pericardium was normal. Coronary angiography was normal (Figure 2). Cardiac magnetic resonance imaging revealed apical akinesia extending to the anterior wall, a T2 hypersignal indicative of myocardial edema. There was a nodular heterogeneous contrast, sparing the sub-endocardial layers on the late enhancement sequences. The diagnosis of myocarditis was made. There were no abnormalities on first-pass perfusion sequences (Figures 3-5). Initially, the patient had received antithrombotic and analgesic treatment with the pain subsiding. He was discharged after eight days of hospitalization with a decrease of the subepicardial lesion (Figure 5) on the electrocardiogram and an improvement of his systolic left ventricular function on Doppler echocardiography. He is currently under daily dose of $5 \mathrm{mg}$ of Bisoprolol and $5 \mathrm{mg}$ Perindopril.

Case 2: A 22-year-old, who has been a professional footballer for the past 3 years, suffering from work-related stress, with no history of smoking or consumption of cocaine or other drugs. There was no significant personal or family history. He was admitted to the cardiac intensive care unit for an intense, constrictive, precordial pain with no radiation, which started 15 minutes after a football match and lasting 10 hours prior to presentation. His blood pressure was 110/80 $\mathrm{mmHg}$, heart rate of 58 beats per minute, respiratory rate of 22 cycles per minute and a temperature of $37^{\circ} \mathrm{C}$. Physical examination was normal. Laboratory analyses showed an elevated troponin I level at $1.95 \mathrm{ng} / \mathrm{l}$ (normal $<0.3 \mathrm{ng} / \mathrm{l}$ ) and CPK-MB level at $98 \mathrm{ng} / \mathrm{ml}$ (normal $<25 \mathrm{ng} / \mathrm{ml}$ ). There was a leukocytosis at $16,670 / \mathrm{mm}^{3}$ with a neutrophilic predominance. Fasting blood glucose, lipid profile and renal function were normal. ECG inscribed a regular sinus rhythm with a heart rate of 58 cycles per minute, a complete right bundle branch block, extensive anterior necrosis, sub-epicardial lesion in the apico-lateral leads and an isolated ventricular premature beat with a full compensatory pause (Figure 1). 


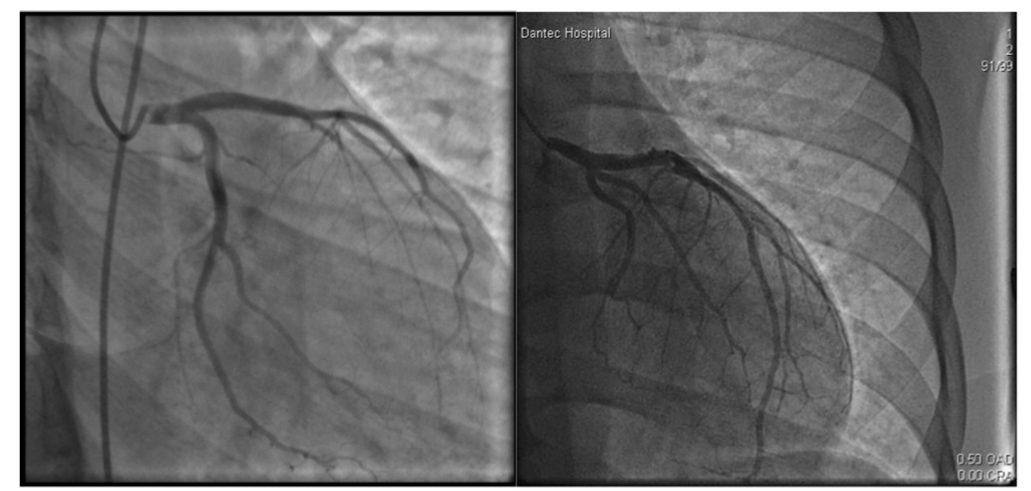

LAO (left anterior oblique view)

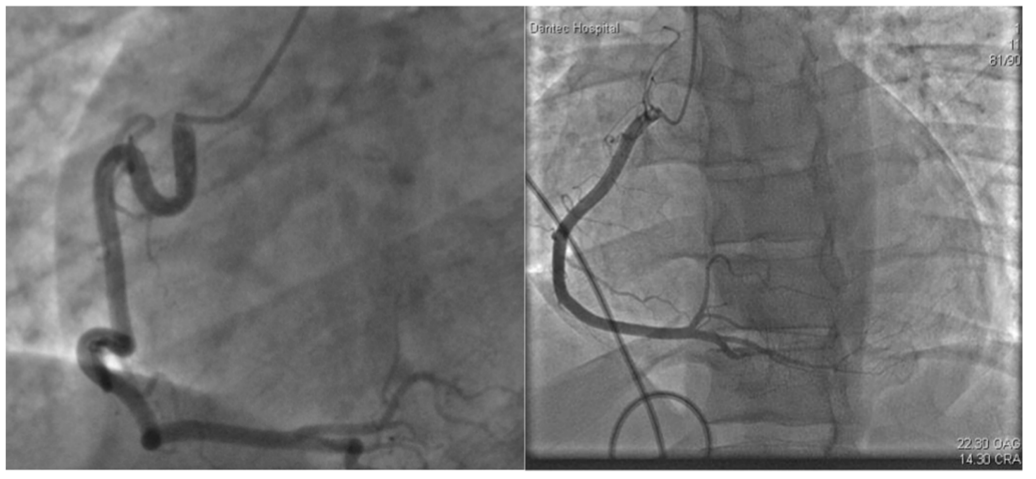

RAO (right anterior oblique view)

Figure 2. Coronarography of the two patients showing no anomaly of the left and right coronary arteries (Left: First patient; Right: Second patient).

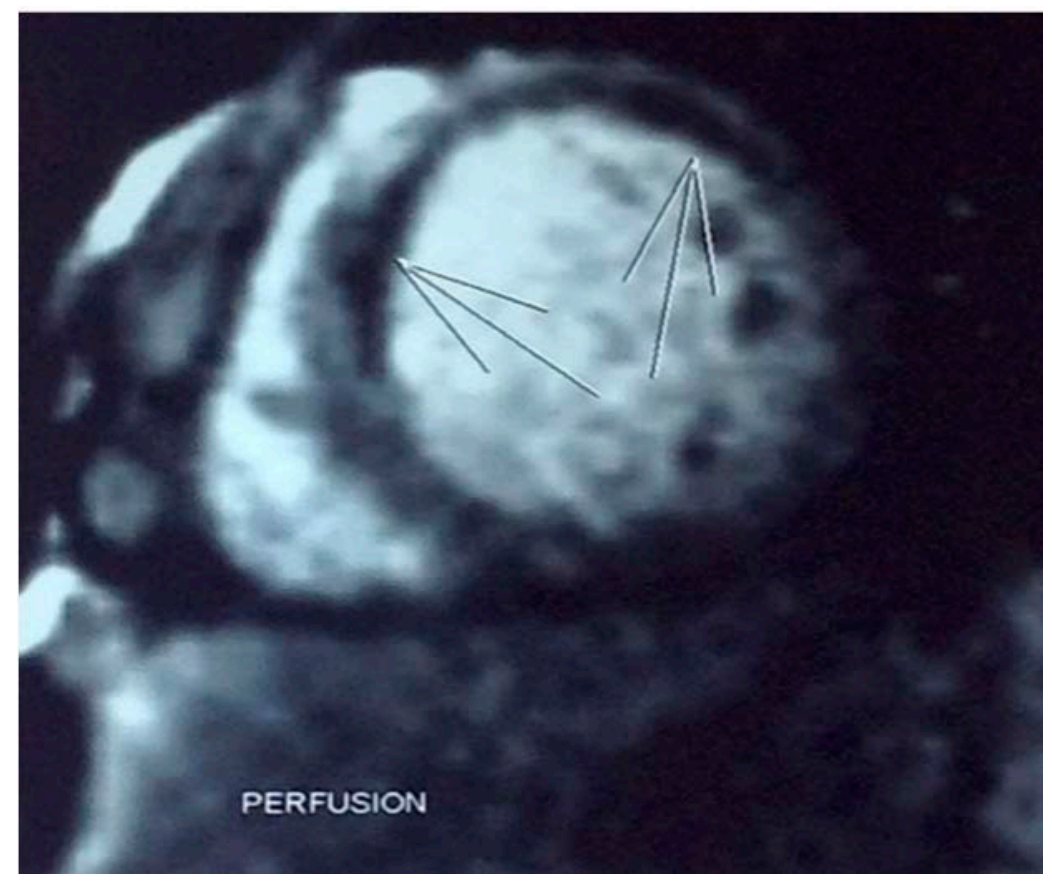

Figure 3. Cardiac magnetic resonance imaging of the second patient on firstpassage perfusion (Arrow: a delayed apical anterior enhancement). 


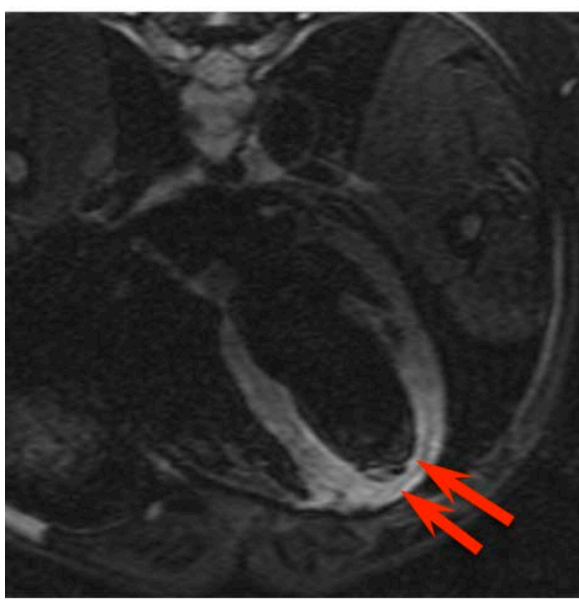

(a)

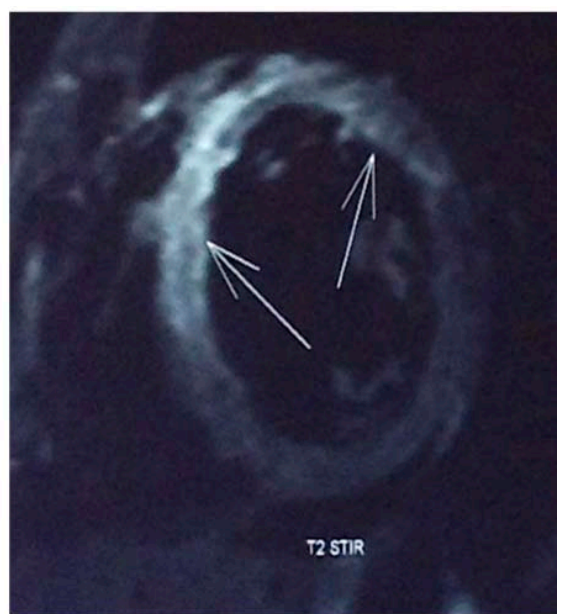

(b)

Figure 4. Cardiac magnetic resonance imaging of the two patients, sequence black Blood, T2-weighted ((a) First patient, 4 chambers view; (b) Second patient, short axis view; Arrow: Hyperintense signal witnessed a myocardial edema at the apical anterior wall of the left ventricle).

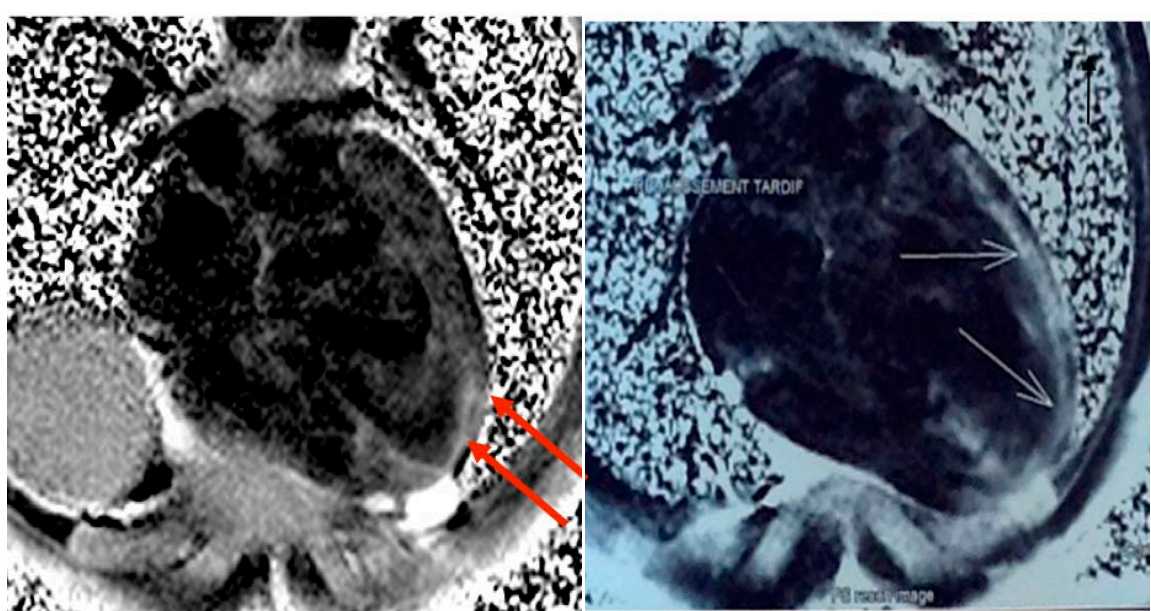

(a)

(b)

Figure 5. Cardiac magnetic resonance imaging of the two patients, sequence "T1 inversion recovery", 4 chambers view ((a) First patient: Delayed enhancement respecting the subendocardial layers; (b) Second patient: Quasi-delayed transmural enhancement of the left ventricle).

Chest x-ray was normal. Transthoracic Doppler echocardiography showed left ventricular wall motion abnormalities in the antero-septo-medial and apical walls, moderate systolic dysfunction of the left ventricle at $45 \%$, a diastolic dysfunction with elevated filling pressures and an adherent apical thrombus of 1.8 $\mathrm{cm}^{2}$ (Figure 6). There was no apical ballooning of the left ventricle. Cardiac MRI done on the fifth day of admission revealed a pattern in favor of an acute antero-apical infarction with severe hypokinesia and quasi-transmural late enhancement, extending to the anterior papillary muscle of the mitral valve and reduction of LVEF at 33\% (Figures 3-5). Coronary angiogram was normal 
(Figure 2). The patient received anti-thrombotic and analgesic treatment with remarkable improvement of symptoms. There was no complication and he was discharged after 12 days of hospitalization with a regression of the subepicardial lesion on the ECG (Figure 7) and a disappearance of the intraventricular thrombus but a persistence of systolic dysfunction on transthoracic Doppler echocardiography. He is currently receiving a daily dose of $100 \mathrm{mg}$ of aspirin, 75 $\mathrm{mg}$ of clopidogrel, $2 \mathrm{mg}$ of acenocoumarol, $5 \mathrm{mg}$ of bisoprolol, $10 \mathrm{mg}$ of ramipril and $80 \mathrm{mg}$ of atorvastatin.

\section{Discussion}

Cardiac MRI is currently the key diagnostic test in acute myocarditis [2]. It also plays a major role in the secondary exploration of ischemic heart disease, particularly myocardial infarction [3].

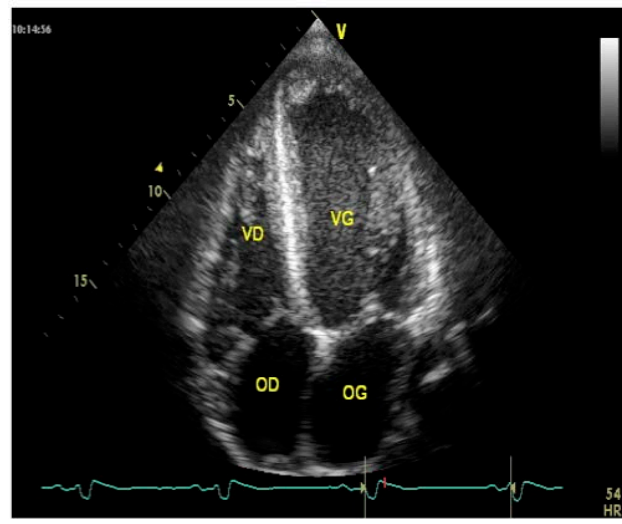

(a)

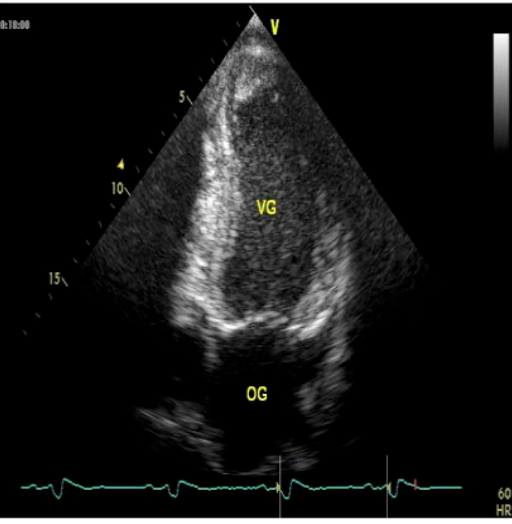

(b)

Figure 6. Second patient transthoracic echocardiogram showing a thrombus and spontaneous contrast in the left ventricle ((a) Apical 4 chamber view; (b) Apical 2 chamber view).

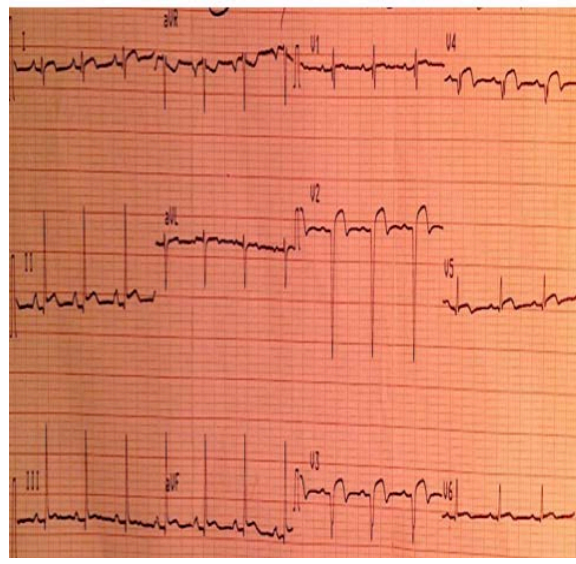

(a)

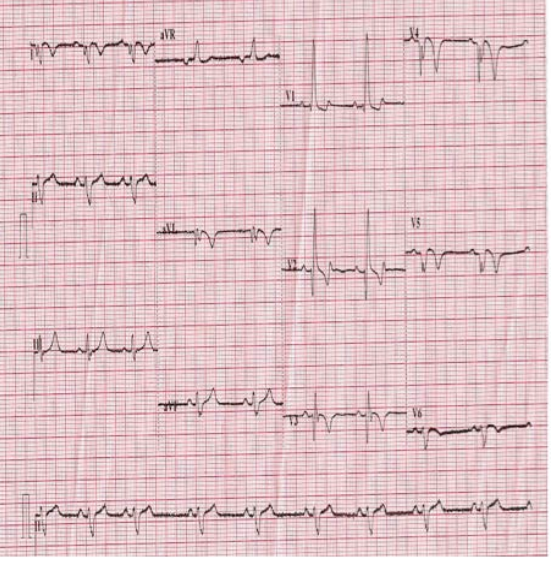

(b)

Figure 7. Electrocardiogram at discharge of the two patients showing persistence of primary repolarization changes ((a) First patient: regression of subepicardial lesion; (b) Second patient: accentuation of subepicardial ischemia in apical lateral territory). 
In our report, we have two young subjects presenting with precordial pain, elevated troponin level, ECG signs of coronary insufficiency and segmental wall motion abnormalities of the left ventricle. Coronary angiography was normal in both patients.

In case 1, there was an antecedent of febrile syndrome as well as tobacco consumption until 3 months prior to presentation [4]. Although the diagnosis of myocardial infarction was most likely, it was ruled out by cardiac MRI, which concluded with a diagnosis of acute myocarditis with apical and anterior wall akinesia and a nodular heterogeneous contrast enhancement sparing the sub-endocardial layers on the enhancement sequences (Figures 3-5).

In case 2, we found him to have significant work-related stress [5] with repeated ECGs showing features similar to those at admission. The diagnosis of myocarditis was posed but ruled out by MRI, which demonstrated a lack of perfusion on the first pass sequences, a quasi-transmural late enhancement of the anterior and apical walls of the left ventricle (Figures 3-5).

This demonstrates the importance of MRI as a useful, accessible non-invasive tool in making the differential diagnosis between these two pathologies, which may sometimes present with a similar clinical picture [1] [6]. This diagnostic tool helps to provide accurate diagnosis of chest pain associated with elevated cardiac enzymes [3] [7] [8].

Four sequences are mainly studied to assess perfusion and myocardial viability [7] [9].

In the context of myocarditis, cardiac MRI is a key diagnostic tool [2] [6] described for the first time by Friedrich [10].

In the "cine" sequence, the affected segments may exhibit kinetic disorders of variable importance [9]. In the beginning (first 5 days), we often find on the "black blood, weighted T2" sequences, a hypersignal corresponding to edema with sometimes a pericardial effusion [9]. After injection of gadolinium, a homogeneous early enhancement over the entire myocardium is found on the perfusion sequences. Ten minutes later, the viability sequence shows a non-systematic diffuse or nodular sub-epicardial enhancement [9]. In the context of myocardial infarction, in the cine sequence, there are disorders of segmental kinetics consistent with enhancement [9] [11] [12] [13].

Myocardial perfusion is studied in first pass perfusion sequence during the first passage of gadolinium. If there is a lack of perfusion of a myocardial territory linked to the occlusion of a coronary artery (no-reflow), there is a lack of enhancement of importance proportional to the concerned territory [12].

In the viability sequence, the existence of a late enhancement characterized by a well-defined sub-endocardial or even transmural hypersignal translates to an infarcted and fibrotic myocardium [3]. The importance of enhancement is also a prognostic factor; it would thus make it possible to predict the functional recovery of the infarcted myocardium [7] [9].

Tissue characterization by cardiovascular magnetic resonance imaging provided a good diagnostic yield in this large clinical population of patients with sus- 
pected AM. It provided incremental diagnostic value by ruling out the diagnosis of acute myocarditis on one hand and by potentially sparing acute myocarditis patients from CAG on the other [14].

These cases illustrate the value of MRI in differentiating acute MI from myocarditis in this young patient with atypical chest pain. Further, it demonstrates the value of MRI in detecting previous MIs and reinforces the importance of searching for precipitants of MI in young patients [15].

\section{Conclusion}

These cases exhibit the use of cardiac magnetic resonance imaging for diagnosis in such scenarios as often if the angiogram is normal. Cardiac MRI is a diagnostic tool that helps to differentiate between myocardial infarction and myocarditis in its pseudo-infarctoid form, especially when the context is unclear. It also makes it possible to evaluate the prognosis.

\section{References}

[1] Casolo, G., Minneci, S., Manta, R., et al. (2006) Identification of the Ischemic Etiology of Heart Failure by Cardiovascular Magnetic Resonance Imaging: Diagnostic Accuracy of Late Gadolinium Enhancement. American Heart Journal, 151, 101-108. https://doi.org/10.1016/j.ahj.2005.03.068

[2] Croisille, P. (2004) Cardiopathies ischémiques (perfusion myocardique et viabilité): Techniques et résultats. Journal de Radiologie, 85, 1811-1818. https://doi.org/10.1016/S0221-0363(04)97752-9

[3] Dec Jr., G.W., Waldman, H., Southern, J., et al. (1992) Viral Myocarditis Mimicking Acute Myocardial Infarction. Journal of the American College of Cardiology, 20, 85-89. https://doi.org/10.1016/0735-1097(92)90141-9

[4] Deux, J.F., Garot, J., Jourdan, G., et al. (2007) IRM des cardiopathies ischémiques. Feuillets de Radiology, 47, 315-330. https://doi.org/10.1016/S0181-9801(07)73229-3

[5] Friedrich, M.G., Strohm, O., Schulz-Menger, J., et al. (1998) Contrast Media-Enhanced Magnetic Resonance Imaging Visualizes Myocardial Changes in the Course of Viral Myocarditis. Circulation, 97, 1802-1809. https://doi.org/10.1161/01.CIR.97.18.1802

[6] Joussein-Remacle, S., Delarche, N., Bader, H., et al. (2006) Facteurs de risque de l'infarctus du myocarde du sujet jeune: Registre prospectif sur un an. Annales de Cardiologie et d' Angéiologie (Paris), 55, 204-209. https://doi.org/10.1016/j.ancard.2006.05.001

[7] Labeyrie, M.A., Marmurzstein, J., Daoud, B., et al. (2008) Apport de la tomodensitométrie coronaire et de l'IRM cardiaque dans le syndrome coronaire aigu. La Presse Médicale, 37, 705-715. https://doi.org/10.1016/j.lpm.2007.07.038

[8] Laissy, J.P., Messin, B., Varenne, O., et al. (2002) MRI of Acute Myocarditis: A Comprehensive Approach Based on Various Imaging Sequences. Chest, 122, 1638 1648. https://doi.org/10.1378/chest.122.5.1638

[9] Leurent, G., Langella, B., Boulmier, D., et al. (2008) Apport de l'IRM cardiaque dans le diagnostic étiologique des syndromes douloureux thoraciques à réseau coronaire angiographiquement normal. Annales de Cardiologie et d' Angéiologie (Paris), 57, 109-115. https://doi.org/10.1016/j.ancard.2008.02.002

[10] Mahrholdt, H., Goedecke, C., Wagner, A., et al. (2004) Cardiovascular Magnetic 
Resonance Assessment of Human Myocarditis: A Comparison to Histology and Molecular Pathology. Circulation, 109, 1250-1258.

https://doi.org/10.1161/01.CIR.0000118493.13323.81

[11] Monney, P.A., Sekhri, N., Burchell, T., et al. (2011) Acute Myocarditis Presenting as Acute Coronary Syndrome: Role of Early Cardiac Magnetic Resonance in Its Diagnosis. Heart, 97, 1312-1318. https://doi.org/10.1136/hrt.2010.204818

[12] Rodière, M., Jankowski, A., Thony, F., et al. (2007) Apport de l'IRM cardiaque à l'exploration des cardiopathies ischémiques. Médecine Nucléaire: Imagerie Fonctionnelle et Métabolique, 31, 597-603. https://doi.org/10.1016/j.mednuc.2007.09.003

[13] Sarr, M., Ba, D.M., Ndiaye, M.B., et al. (2013) Acute Coronary Syndrome in Young Sub-Saharan Africans: A Prospective Study of 21 Cases. BMC Cardiovascular Disorders, 13, 118. https://doi.org/10.1186/1471-2261-13-118

[14] Biesbroek, P.S., Hirsch, A., Zweerink, A., et al. (2017) Additional Diagnostic Value of CMR to the European Society of Cardiology (ESC) Position Statement Criteria in a Large Clinical Population of Patients with Suspected Myocarditis. European Heart Journal-Cardiovascular Imaging, jex308. https://doi.org/10.1093/ehjci/jex308

[15] Walsh, J.L., Harris, B.H.L., Gharzuddine, W., et al. (2017) Myocardial Infarction Masquerading as Myocarditis in a Patient with Factor V Leiden: Unmasked with MR. BMJ Case Reports. https://doi.org/10.1136/bcr-2017-220652 